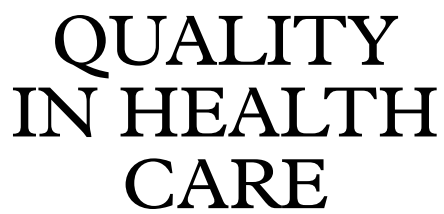

Editorials

\title{
Quality improvement in primary health care: a European perspective
}

Primary health care in different countries is facing a common and important set of challenges. Three papers reporting quality improvement initiatives in primary health care are included in this issue of the fournal, and they highlight progress that has been made in recent years. ${ }^{1-3}$

One explanation for the creation of quality improvement systems in primary health care in most European countries lies in the concern about rising healthcare costs and the belief that a renewed emphasis on primary care could be one solution. ${ }^{4}$ Measures to improve the efficiency of primary care have the potential to reduce the referral of patients to more expensive secondary care. It might also be possible to transfer some elements of care from hospitals to the community. However, if such changes are to be introduced successfully, primary health care requires modification, and even modernisation. Thus, quality improvement in primary health care has a dual function-to improve the quality of care and also to support the development of a service that can take on a wider range of responsibilities.

One characteristic of primary health care that presents an obstacle to development is its fragmented structure. It is provided by individual professionals or small teams housed in widely dispersed practices. This can lead to isolation, even among practices in cities, and isolation can in turn give rise to delay in implementing appropriate clinical care, wide variations in performance, and even idiosyncratic and unacceptable patterns of care. Practice visiting is one approach to tackle this problem, and Eliasson and colleagues present an example from Sweden. ${ }^{1}$

Primary health care in Sweden is comparatively well resourced, and generally provided by multidisciplinary teams. However, in a large country with a relatively small population, isolation could be a problem. The main objectives of the scheme were to enable primary healthcare teams to discover their strengths and weaknesses, and to encourage them to extend their quality improvement activities. The procedure involves the collection of data about clinical and other aspects of performance, and uses standard tools developed for use throughout the country.

In Germany the system of primary health care is less formalised, as various specialists may offer primary care and general practitioners do not act as gatekeepers to secondary care. Solo practice predominates, and therefore strategies for overcoming isolation are particularly important. Gerlach and Beyer ${ }^{2}$ report such a strategy in which professionals have been invited to join quality circles that meet regularly and undertake various activities, including peer review, and comparison of data about performance. The groups are led by a trained moderator.

Another obstacle that may delay the development of primary health care is poor coordination between primary and secondary care. A new approach to this problem is reported by Olesen et $a l^{3}{ }^{3}$ General practitioners in Denmark have been appointed as advisers to hospital departments to improve communication and the implementation of shared protocols. The value attached to this programme by health service managers is shown in their agreement to fund general practitioners to fulfill this role.

These examples of quality improvement in European primary health care raise two important issues. Firstly, health systems throughout Europe are facing similar problems, and therefore there may be much that we can learn from each other. Practice visiting or quality circles may be appropriate for many countries as well as Sweden or Germany. General practitioner advisers in hospitals may be of value in other countries as well as Denmark, but to ensure that new methods are fully evaluated, and implemented if cost effective, convincing research evidence is necessary. It may be possible in one country to develop a new method of quality improvement, but its formal evaluation may be prevented by its early adoption. Therefore, links are required between researchers and funders in different countries to enable formal studies to be organised elsewhere.

The second issue concerns the nature of primary health care itself. To what extent can it be developed to take on some of the functions of secondary care without putting its traditional role at risk? A core feature which underlies the attitudes of many patients towards primary care is the provision of personal care, ${ }^{5}$ but in an extended primary healthcare service personal care could be sacrificed for efficiency. In reforming primary health care, quality improvement has an additional essential task - to monitor and maintain the level of personal care in accordance with the wishes of patients.

RICHARD BAKER

Director, Eli Lilly National Clinical Audit Centre, Department of General Practice and Primary Health Care, University of Leicester, Leicester General Hospital, Gwendolen Road, Leicester LE5 4PW, UK

1 Eliasson G, Berg L, Carlsson P, et al. Facilitating quality improvement in primary health care by practice visiting. Quality in Health Care 1998;7:48-54.

2 Gerlach FM, Beyer M. A new concept for continuous documentation and development of quality circle in ambulatory care: initial results from an information system in Germany. Quality in Health Care 1998;7:55-60.

3 Oleson F, Jensen PB, Henriksen LS. General practitioners as advisers and co-ordinators in hospitals. Quality in Health Care 1998;7:42-47.

4 Grol R, Baker R, Roberts R, et al. Systems for quality improvement in general practice. A survey in 26 countries. European fournal of General Practice 1997;3:65-8.

5 Baker R. A pragmatic model of patient satisfaction in general practice: progress towards a theory. Quality in Health Care 1997;6:201-4. 


\section{Where does clinical audit fit in?}

The new United Kingdom government has already produced a paper-The new NHS: modern dependablewhich outlines the future shape and direction that the government wish to give to the United Kingdom National Health Service (NHS). One of the proposals within this paper is the establishment of a National Institute for Clinical Effectiveness as a key feature of the drive for improved quality in clinical practice. ${ }^{1}$ This follows other initiatives to improve the quality of health care including the introduction of clinical audit as part of the last government's white paper on the NHS in 1989. We can therefore expect renewed interest in the process of clinical audit. Health authorities responsible for the disbursement of considerable sums of money for audit are already beginning to ask how they can ensure better value for money. All of this makes the paper in this issue Why is the evaluation of the cost effectiveness of audit so difficult? particularly timely. ${ }^{2}$ The fact that it bravely reports negative findings makes it none the less valuable, for it raises several important questions. Unlike many audit topics the one chosen by the authorsthe use of intravenous thrombolysis for patients with suspected acute myocardial infarction-seemed relatively clear cut. The evidence for the effectiveness of the procedure had been confirmed by several large trials, and the outcome measure chosen-namely, the extra number of cases treated in the participating hospitals-nonproblematic. But the disappointing results obtained lead the authors to ask whether they had properly controlled for factors, other than clinical audit, which could have influenced behaviour. To my mind the answer must be yes, and the experience with this particular audit suggests not only that questions about cost effectiveness may at this stage be premature, but that the process of audit has to be seen in the broader framework of organisational analysis.

The formal definition of clinical audit is as a "professionally led initiative which seeks to improve the outcome of patient care as a result of clinicians examining their practice and modifying it appropriately". As currently interpreted audit involves drawing a boundary around a procedure (or set of procedures) and isolating it for examination from the complex totality of the organisation in which it is occurring. The desirable modification of practice which should ensue might involve any of the following: a change of technique (as in surgery); a modification of a drug regime; or a change in the criteria for patient treatment. But whether or not desirable modifications occur will be facilitated or hampered by a host of wider organisational features, many of which will lie beyond the boundary of the particular audit. Audit is concerned with change, and studies of organisational change can tell us much about the ease or difficulty with which the specific outcome of audit can be effected and sustained. Firstly, change is more likely to be accepted when the knowledge base for audit is widely agreed and not contested by participants, or if its problematic character can be acknowledged without threat to the status of participants. Secondly, the lessons of audit will be more easily accepted if they do not require a change in role definition (particularly important if different professional groups are involved), and when role modification is required, the consequences are fully worked through. Thirdly, the adoption of the lessons of audit may require change in methods and relations beyond the immediate audit task-say in administrative procedures. Failure to recognise this is likely to produce unhelpful tensions.
Fourthly, a learning culture in the organisation as a whole will not only support participation in audit, but will facilitate the early adoption of new methods and their sustained application.

Of course audit is not the only tool available for improving clinical effectiveness. We have protocols and guidelines, a restructuring of education and training to place emphasis upon the importance of evidence, and even greater attention to the implementation of research findings in practice (the drive for evidence based medicine). However, there remains a considerable amount of dissatisfaction with the progress that has been made. It is not difficult to find glaring examples of failure to adopt well established best practice. Some of these can be attributed to individual idiosyncrasies, but more often they can be explained by failures to understand the complexities of organisational processes. And here much can be learned from the now considerable body of knowledge of non-medical organisations. This is not to deny the quite distinctive characteristics of organisations the task of which is the delivery of health care within the NHS. To begin with all NHS organisations have extremely close links with the wider society, both at the political level and by virtue of the tasks which they exist to perform. Hospitals and general practice are "people processing organisations", an inelegant phrase which, however, serves to underline the fact that the people to whom health care is being delivered become in some way part of the organisation, and are individual patients not only with an immense variety of medical conditions but of attitudes and expectations. Another feature of medical organisations is the indispensability of professionalsdoctors, nurses, professionals in diciplines supplementary to medicine, technicians - to the carrying out of the care activity and hence the central position of professional knowledge. However, it is a body of knowledge which remains, despite rapid technical advances, a mix of science and craft skills.

The special feature of the medical domain does not undermine the relevance of the more general body of organisational analysis from which certain relatively simple themes emerge. Modern organisations are highly complex systems based on complex division of labour which then require elaborate coordination and control systems. Both the nature of this complexity and the environment in which organisations operate generate a high degree of uncertainty. They are interactive systems so that change in one area will have repercussions for the rest of the system. The work performed in organisations is the responsibility of people who will have varying attitudes and values, aspirations, and expectations, and the extent to which these things are shared varies. There is no one best way to organise but the successful institution will be one which searches constantly for the best fit between the nature of the task, the demands of the environment, and the values and aspirations of its employees.

If the huge changes in the NHS envisaged in the white paper are to be achieved successfully an analytical framework such as this needs to be applied at every level.

\section{DOROTHY WEDDERBURN}

Emeritus Professor of Industrial Sociology, Imperial College of Science, Technology and Medicine, London, UK

\footnotetext{
1 The new NHS - modern dependable. London: The Stationary Office, 1997. (Cm 3807.)

2 Robinson MB, Thompson E, Black NA. Why is evaluation of the cost effectiveness of audit so difficult? The example of thrombolysis for suspected
tiventions acute myocardial infarction. Quality in Health Care 1998;7:19-26.
} 


\section{Nursing: quality in numbers}

In the modern health service nurses currently make up about $70 \%$ of the workforce, they cost $40 \%$ of total health service expenditure, $60 \%$ of the total health service pay bill, and they command $3 \%$ of the public purse. Therefore, it is not unreasonable for some people to question whether a rich skill mix of mostly qualified staff gives a higher quality of care than a cheaper skill mix of mostly unqualified staff.

This question has led to other equally important questions, including: are there enough nurses working with the right patients, in the right place, at the right time, doing the right thing, in the right way? These questions will continue to taunt the nursing profession and health services managers in the next millennium when the creation of a flexible, effective, and efficient nursing workforce in the face of change will remain a major priority.

Throughout the 1990s the replacement of registered nurses with unskilled support staff has been a common occurrence in the United Kingdom, Australia, and the United States. This has caused consternation among nurses and fuelled the belief (either rightly or wrongly) that skill mix reviews are merely excuses for skill substitution exercises. Such initiatives have had a predictable and detrimental effect on the quality of care. Unqualified support staff have a smaller repertoire of functioning skills and trained independent judgement. Unlike registered nurses they can only perform a narrow range of procedures and therefore have limits to their ability. It is also evident that an increase in their numbers has the effect of registered nurses spending less time in direct patient care; rather, time is spent inducting, supervising, teaching, and directing these unqualified support workers.

It seems logical, therefore, that increasing the numbers of unqualified staff could ultimately lead to low productivity of all staff and as a result higher costs. However, although this apparent lack of productivity may be compensated by support workers' lower appointment costs, lower salary, and lower training costs, it is clear that they do not possess the basic theoretical foundation for competent practice. Therefore, they are more likely to use task focused physical care based on ritual and routine.

Evidence shows that a rich skill mix of qualified nurses in direct contact with patients was much more likely to deal with the total needs of the patient than fewer numbers of nurses acting to give care through support workers. ${ }^{1}$ This allows each nurse to function in a full professional role spending more time working with patients and less time in the supervision of unqualified staff.

Replacing qualified nurses with unqualified staff also leads to low morale among the qualified group, which in turn leads to higher costs and lower quality of care. ${ }^{2}$ In his book on quality for health service managers Ovretveit argued that reductions in the number of qualified staff means more work for the remaining staff, more dissatisfaction among staff and patients, higher levels of casual sickness and absenteeism, increased costs, and ultimately more staff cuts. ${ }^{2}$ The presence of this low morale cycle does not give the impression of valuing staff, one of the hallmarks of total quality management.

A management myth exists based on the proposition that although the economic crunch has led to a reduction in the quantity of nursing staff-quality of care has not suffered. Donabedian disputes the basic premise of this. He points out that: "It should be clear to everyone, though sometimes we seem to forget it, that more quality must cost more: in . . . resources . . knowledge, skill, time, attention, dedication. Being so precious it does not come cheaply". ${ }^{3}$

I undertook a comprehensive literature review and uncovered over 60 studies focusing on skill mix in nursing. All but four indicated that a rich skill mix of mostly qualified staff has a positive effect on quality of patient care. Summarising these studies it is clear that nurse staffing levels and skill mix make a difference to the outcomes of patients in hospital. By reducing mortalities, duration of stay, complications, and increasing patient satisfaction and patient readiness and ability to function on discharge, qualified nurses contribute to both the quality of service and the containment of costs. ${ }^{4}$

More recently the results from two new studies from the United States found that the more qualified the nurses the lower the rates of adverse outcomes - such as errors in administration of medication, patient falls, decubiti, nosocomial infections, and cardiopulmonary arrests. ${ }^{56}$ Qualified staff also have a positive effect on a range of care interventions such as prevention of stress, promotion of wound healing, rehabilitation from serious physical problems, and continence care. ${ }^{7}$ Unlike untrained novices, the expert qualified nurse can detect minute, almost imperceptible changes in the patients' condition. These observations can be life saving in many instances and there are obvious quality implications to the early detection of side effects and complications.

The cost savings related to such processes and outcomes are considerable. Qualified nurses can save money: by reducing overstocking, by reducing the number of unnecessary procedures, and by preventing the purchase of inappropriate products. There is robust evidence to indicate that in those hospitals that increased their numbers of registered nurses the labour costs as a percentage of total hospital expenditure declined. ${ }^{4}$ It can be argued that the number of qualified nurses is not causing an increase in healthcare expenditure, rather it is the purchase of new drugs, technology, and equipment that are pushing up the costs.

Two excellent papers in this issue of Quality in Health Care analyse the contribution that qualified nurses make to a quality health service. The first discusses the successful role that nurses are playing in clinical audit and identifies several factors that impede further success. ${ }^{8}$ The second paper highlights the importance of an appropriate nursing skill mix with the authors indicating the favourable impact that qualified nurses have on inpatient mortalities. They also provide evidence of the detrimental effect that non-supportive and inflexible organisations have on nurse job satisfaction. ${ }^{9}$

The reader would be forgiven for concluding from this editorial that managers who call for skill substitutions were interested in cost and not quality and nurses were interested in quality and not cost. This conclusion is too simplistic in an area where quality is difficult to measure and most of the important effects of qualified nurses are invisible to the naked eye.

However, there are some things that can be stated with a degree of certainty. If the time spent by qualified staff in direct patient care is reduced due to their induction, supervision, and teaching of unqualified staff, then any salary savings will be off set by a decrease in productivity. Also, if the quality of care declines as the richness of the skill mix 
declines any efficiency savings may be costly in terms of deterioration in clinical effectiveness.

HUGH MCKENNA

Professor of Nursing

University of Ulster at fordanstown, Newtonabbey, County Antrim, Northern Ireland BT37 OQB, UK.

1 McKenna HP. Skill mix substitution and quality of care: an exploration of assumptions from the research literature. $\mathcal{F}$ Adv Nurs 1995;21:452-9.

2 Ovretveit J. Health services quality. London: Blackwell, 1992.
3 Donabedian A. The price of quality and the perplexities of cure. Chicago: Centre for Health Administration Studies, University of Chicago, 1986.

4 Prescott PA. Nursing: an important component of hospital survival under a reformed health care system. Nursing Economics 1993;11;192-9.

5 Blegen M. Research on the use of unlicenced assistants. Personal communication through the Nurse Res List on Internet, 1997.

6 Aiten I. Lower medical mortality among a set of hospitals known for good nursing care. Philadelphia: University of Philadelphia, 1994

7 Wilson-Barnett J. Effectiveness of nursing [editorial]. Fournal of Nursing Management. 1995;3:285-6.

8 Cheater FM, Keane M. Nurses' participation in audit: a regional study. Quality in Health Care 1998;6:27-36.

9 McKee M, Aiken L, Rafferty AM, et al. Organisational change and quality of health care: an evolving international agenda. Quality in Health Care 1998;6:37-41. 